\title{
Variables psicosociales vinculadas con la satisfacción laboral
}

\section{María Fernanda Zúñiga Arias}

\section{Universidad de Iberoamérica}

\section{Resumen}

El presente estudio muestra un acercamiento real al trabajo que realizan día a día cinco trabajadores operativos de un gobierno local, se profundizó en el análisis de su historia laboral determinando los principales factores psicosociales y cómo estos permiten potenciar la satisfacción laboral en los trabajadores. Los análisis se realizaron a través de un método hermenéutico-dialéctico, enfocándose en las emociones y pensamientos sobre la realidad de los trabajadores en su ambiente laboral

Palabras clave: historia laboral, estigmatización, motivación, actitud hacia el trabajo

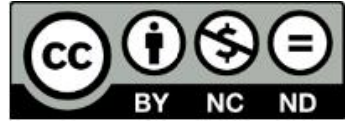




\section{Introducción}

De acuerdo con Furham (2009) la organización es un lugar de trabajo en el cual las personas le dedican tiempo y esfuerzo. Asimismo, la organización es un sistema social, con creencias, valores, cultura y comportamientos propios (Palací, 2008) por lo que en el presente estudio se aprecia como estos cinco trabajadores (cuatro Aseo de Vías y uno de Cementerio) han enfrentado situaciones laborales y sociales que influyen en su sentir y pensar con respecto al trabajo que realizan.

Estos trabajadores operativos les corresponde ejercer sus funciones en las calles lo cual implica que su interacción no sea solo con la institución sino también con los ciudadanos, pues estos últimos son los que ven a los empleados trabajar día a día en las calles, por lo tanto incluir estos aspectos psicosociales brindan datos para analizar las etiquetas que son puestas por el tipo de trabajo que realizan y relacionarlas con las satisfacción laboral que presentan estos funcionarios.

En la revisión empírica-teórica de investigaciones previas, no hay abundancia de estudios que se dirijan abordar las implicaciones emocionales y sociales que resultan de realizar trabajos operativos como los mencionados anteriormente, por lo que este estudio abre un espacio de conocimiento en este tipo de población y expande el conocimiento psicológico ante lo laboral y social.

En el 2012 se realizó un diagnóstico de clima organizacional en el gobierno local al que pertenecen estos trabajadores, en donde uno de los factores evaluados fue la satisfacción laboral. Ante la insatisfacción de los funcionarios en el trabajo grupal que se desarrolló para dicho diagnóstico, se encontró que se impide el crecimiento personal, hay desvalorización del trabajo mientras que en las estadísticas el 77.5\% puntuó un nivel alto de satisfacción, el $22.1 \%$ puntuó en un nivel medio y finalmente el $0.4 \%$ puntuó un nivel bajo (Alvarado y Zúñiga, 2012).

No habiendo coincidencia entre el trabajo grupal y el instrumento utilizado se infiere que hay presencia de elementos en el ámbito laboral que inciden en el personal negativamente e influyen en la realización del trabajo (Alvarado y Zúñiga, 2012).

Por lo anterior, surge ¿Cuáles son las implicaciones psicológicas y sociales presentes en el quehacer diario de los cinco trabajadores operativos? y ¿Existe una relación entre la satisfacción laboral y los factores psicosociales? 


\section{Aspectos generales de la Psicología Social aplicada a las organizaciones}

De acuerdo con el objeto de estudio de la psicología de las organizaciones, en múltiples ocasiones se ha intentado explicar el comportamiento de la organización aislando al individuo o bien reduciéndolo dentro del fenómeno social. Sin embargo, la organización es un sistema social que está conformada por un grupo de personas, en donde el comportamiento de los individuos influye en la actividad de la organización, siendo esto explicado de la mejor forma por modelos psicológicos. Además, al ser un sistema social emergen situaciones que son producto de las interacciones entre los miembros de la organización y de éstos con la misma institución (Palací, 2008).

Esta interacción en la organización toma lugar en los procesos emergentes, tales como: la toma de decisiones, la comunicación, la motivación, la satisfacción, entre otros. Por lo cual, el aspecto psicosocial articula lo estructural-organizacional con lo individual, con los grupos y con la organización y no se pueden estudiar de forma aislada, sino que deben de ser en conjunto para comprender de forma integral la organización y sus trabajadores (Munduate, 1997 citado por Palací, 2008, p. 25).

Por lo tanto, para conocer la interacción entre el trabajador, la municipalidad y los usuarios, se consideró recolectar la información desde la visión de la realidad de los participantes, teniendo como objetivo general: analizar la historia laboral de los cinco trabajadores operativos en relación a los factores psicosociales influyentes en sus trabajos y la vinculación de éstos factores con la satisfacción laboral, empleando la siguiente línea objetiva de investigación especifica:

Caracterizar el ejercicio laboral de los trabajadores desde su visión de mundo.

Identificar los factores psicológicos y sociales presentes en los cuatro trabajadores de aseo de vías y trabajador de cementerio a partir del trabajo que realizan

Determinar la percepción que tienen estos trabajadores acerca de los factores psicosociales que enfrentan por el ejercicio de su trabajo.

Vincular la influencia que tiene la historia de vida de los trabajadores con la satisfacción hacia el trabajo.

Finalmente, a través de estos objetivos fundamentados, se procedió a desarrollar una investigación que vincula lo laboral y lo social en una población que ha sido poco estudiada y bajo y una metodología estricta, como lo es lo hermenéutico-dialéctica.

\section{Ueso




\section{Factores psicológicos implicados en el trabajo}

Peiró y Prieto (2007) mencionan que la satisfacción está relacionada con las actitudes de los trabajadores hacia aspectos concretos del trabajo tales como la compañía, el trabajo mismo, los compañeros y otros objetos psicológicos del contexto de trabajo.

Por lo tanto, la satisfacción laboral podría concebirse como un concepto subjetivo, no obstante, esta puede ser entendida como el agrado que sienta el trabajador con respecto a las funciones que realiza considerando las dimensiones emocionales, físicas, económicas, sociales (Peiró y Prieto, 2007; Muchinsky, 2007).

Por su parte, la motivación, se entiende como los procesos de carácter individual que impulsan al trabajador actuar mostrando un desempeño y un grado de satisfacción en la organización, ya que los procesos laborales deben dirigirse hacia una misma línea, es decir, trabajador e institución en equilibrio (González \& Olivares, 2009).

Aunado a lo anterior, se destaca la diferencia entre satisfacción y motivación, siendo la primera entendida como la actitud hacia el trabajo y la segunda como el impulso para realizar las labores.

La motivación se puede dividir en extrínseca e intrínseca, la primera se refiere al entorno laboral, es decir, características del ambiente en donde tiene lugar la actividad laboral, tales como: dinero, estabilidad de empleo, oportunidades de ascenso, condiciones de trabajo y el ambiente social. La segunda se entiende como lo contenido del trabajo, es decir son aspectos del trabajo relacionados directamente con el desempeño, y características que posee la actividad del trabajo, estos son: interés, importancia e identificación con el trabajo, autonomía para la realización de la tarea, oportunidad de utilizar conocimientos, habilidades y destrezas (Peiró y Prieto, 2007; Guillén, 2003).

Por su parte, Furham (2009) explica que el comportamiento organizacional constituye todo el comportamiento de un individuo dentro de una organización. Este considera los pensamientos, sentimientos, creencias, actitudes y el desempeño de todos los empleados a través del comportamiento explícito que se muestra en la organización. Es decir, que el comportamiento organizacional se manifiesta de forma general, es lo que se logra percibir en grandes rasgos dentro de una organización. Por tanto, el conocimiento del comportamiento se puede obtener por medio de un estudio sistemático de los procesos individuales, grupales, organizacionales y los procesos de subjetivación implicados en cada uno de estos. 
Por lo anterior, el comportamiento organizacional permite esclarecer cómo funciona la organización y bajo que esquemas cognitivos y conductuales funciona la dinámica organizacional (Furham, 2009) pues esto permitió comprender a mayor profundidad el ejercicio laboral de los trabajadores operativos y como son percibidos estos ante el comportamiento laboral establecido de forma general entre los empleados.

En cuanto a la cultura organizacional, Castro y Lupano (2005) la definen como aquel conjunto de valores y creencias que comparten los miembros de una organización determinada. Estos valores y creencias muestran los lineamientos que sostienen los integrantes acerca de lo que se hace y de lo que se debe hacer dentro de las organizaciones.

\section{Factores sociales implicados en el ámbito laboral}

Se afirma que un individuo y la sociedad no se pueden visualizar de forma separada ya que el ser humano está en un constante crecimiento donde su principal propulsor son las interacciones que establece como persona en instituciones, en tendencias y doctrinas. (Cooley, 1918 citado por Alvarado y Garrido, 2004).

Se infiere que la forma en cómo se desenvuelven las personas tanto en contextos familiares, sociales, educativos, religiosos e institucionales, son fundamentales para establecer estas interacciones que los impulsa a proponerse metas y alcanzar la autorrealización en todas las áreas. Por lo tanto, la forma en que interactúan los trabajadores operativos con sus jefaturas, con los compañeros y los usuarios fuera de la institución influye en su desempeño.

Es decir, al ser los empleados de la Municipalidad que trabajan en las calles implica que la interacción laboral no sea únicamente con la institución, sino también con los usuarios que los ven día a día trabajando en las calles.

Socialmente se ha creado la ideología de que los trabajos como los que realizan Aseo de Vías o personal del Cementerio son trabajos de baja categoría, trabajos que no cualquier persona realizaría, ante una idea como esta Vidal (2002) expone que la estigmatización proviene de atributos desacreditadores hacia las personas, hacia al trabajo o bien hacia grupos sociales, donde se denigra al individuo dentro del contexto social al que pertenece. Es decir, que los trabajadores se les introduce en una categoría social a partir del tipo de trabajo que realicen conllevando a que la sociedad y la organización los defina como personas a partir del ejercicio laboral que realizan.

\section{psico


Asimismo, la persona estigmatizada puede aceptar dichos atributos e inclusive hacerlos parte de su sistema de creencias es decir que "el individuo estigmatizado se define a sí mismo como igual a cualquier otro ser humano, al mismo tiempo que es definido por él mismo y por quienes lo rodean como un individuo marginal" (Goffman, 1963, p. 129 citado por Vidal, 2002, p. 4).

Ante esto no solo se puede dar el estigma hacia las personas, sino que existen una serie de conceptos como: estereotipos, prejuicios y discriminación que puede suscitarse a partir del tipo de trabajo que realicen las personas (Vidal, 2002).

\section{Método}

La presente investigación responde a un estudio de tipo cualitativo, pues comprende la realidad en su situación natural. Hernández, Fernández y Baptista (2003) explican que dentro de este enfoque suele desarrollarse una implicación directa con las experiencias de vida de las personas, favoreciendo la adquisición de un punto de vista desde adentro del fenómeno. No obstante, para alcanzar esta mirada integral es indispensable emplear técnicas de investigación capaces de acoplarse a los requerimientos de la situación.

Por lo tanto, desde este método se comprende los fenómenos sociales desde la propia perspectiva de las personas, desde sus percepciones y significados, es decir, busca abordar el significado y reconstruirlo en el entorno y desde el individuo descubrir los hechos sociales (Palaci, 2008). Con base en esto, se considera que el enfoque metodológico más pertinente sería el hermenéutico-dialéctico, pues éste alcanza un panorama más amplio del contexto de estos trabajadores.

Según Álvarez (2007) se puede centrar en lo que la persona expresa y a partir de esto comprender y extraer una interpretación en base a la información obtenida.

De acuerdo con el análisis hermenéutico-dialéctico se busca conocer la vida psíquica a partir de sus representaciones para descubrir e interpretar lo mejor posible las palabras, los escritos, los gestos, que proporcione la persona durante su relato. Además, este enfoque consiste en un paradigma interpretativo-comprensivo que permite revelar los significados de las realidades y analizarlas para obtener una comprensión de las mismas (Álvarez, 2007).

A cada participante se le asignó una letra (A-B-C-D-E) para salvaguardar la confidencialidad de los datos y exponer sus historias anónimamente. 


\section{Instrumentos}

Se empleó una entrevista a profundidad, la cual se dirigió a la comprensión de la percepción que tienen los sujetos participantes respecto de sus vidas, experiencias o situaciones laborales a través de su discurso. No se trato de una entrevista estructurada de pregunta y respuesta, sino que se empezó a formular preguntas en relación al relato que la persona está brindando. En esta técnica, se estableció un rapport y se formuló una pregunta inicial no directa y conforme se avanzó en el relato, se intentó buscar los intereses de la investigación (Hernández, Fernández y Baptista, 2003; Barrantes, 2007).

Según Barrantes (2007) dentro de las entrevistas a profundidad se encuentran las historias de vida, para efectos de esta investigación se trato de "aprehender las experiencias destacadas del trabajo" de los participantes y las definiciones que aplican a tales experiencias, es decir, fue un relato de la trayectoria laboral de estos trabajadores dentro de la Municipalidad. Con esto se logró poner en escena los hechos a partir del discurso y de la escucha, no obstante para no perder detalle del relato de los participantes se les solicitó permiso para grabar su relato bajo los términos de confidencialidad, los cinco participantes estuvieron de acuerdo, y se firmó bajo un consentimiento informado.

Una vez que se finalizaba con la entrevista a profundidad, se les solicitó a los participantes, completar una frase incompleta, la oración era la siguiente: "No me voy sin decir..." y "Ser trabajador municipal significa para mi...", ya que el fin fue obtener información acerca de la funcionalidad que tuvo la misma y conocer el sentir y pensar de cada uno de los participantes al finalizar la entrevista y cuál es la actitud hacia el trabajo de manera concreta y concisa.

\section{Procedimiento}

La recopilación de la información y proceso de análisis, se siguieron los siguientes pasos:

1. Realización de la entrevista a profundidad: entrevistas semi-estrucutradas y de forma individual. Se elaboró una guía con posibles preguntas pero se facilitó el espacio para que el trabajador contará libremente su historia dentro de la institución.

2. Transcripción y digitación de las entrevistas realizadas, de una hora y media a dos horas, cada una.

\section{psis


3. Análisis del discurso según las categorías de análisis propuestas, estas son: historial laboral inicial, motivación al logro, estigmatización social y actitud hacia el trabajo.

4. Integración del análisis según lo hermenéutico-dialectico, siguiendo: la intención, el significado, la función y el condicionamiento de la acción, interpretado a partir del discurso de los trabajadores.

Lo hermenéutico-dialectico y sus cuatro pasos para integrar el análisis, se basaron en los siguientes fundamentos teóricos, expuestos por Álvarez (2007):

1. Intención: filosofía de vida que presenta el trabajador municipal con respecto a su trabajo

2. Significado de la acción: interpretación del contexto y la situaciones concretas que se presentan en el trabajo de los participantes

3. Función: responde a lo latente, es decir, lo que no está explicito en el proceso, mediante la identificación de gestos e inconsistencias entre el "decir y el hacer"

4. Condicionamiento ambiental y cultural: representa un contexto que define al individuo dentro de su realidad social, desde un conjunto de costumbres, normas, patrones de conducta, valores, modos de ver y pensar de la vida, de enfocar los problemas y las soluciones, entre otros.

Luego de lograr la identificación de estos elementos, fue posible comprender la lógica interna del fenómeno para hallar la comprensibilidad de los hechos en relación con la totalidad, es decir, se logró interpretar cómo el trabajador operativo municipal construye su propia realidad en el trabajo, los factores psicosociales presentes y la vinculación de estos con la actitud hacia el trabajo (satisfacción).

\section{Resultados}

Desde la percepción de los trabajadores de Aseo de Vías y el trabajador del Cementerio, representan ser cinco personas que tienen una historia, una razón para ser quiénes son y trabajar en lo que trabajan. Al paso de los años han enfrentado situaciones desagradables y agradables que los han marcado emocionalmente pero que han asumido como parte del trabajo y han buscado una motivación para seguir adelante. 
El tipo de trabajo que realizan es fuera de la institución a excepción del trabajador del cementerio, pero han sido trabajos que socialmente se han considerado de baja categoría y se les ha atribuido una serie de estereotipos, prejuicios y discriminaciones, es decir, son trabajos que han sido estigmatizados socialmente y que los mismos trabajadores han mencionado (Vidal, 2002).

Considerando que los factores psicosociales en el trabajo son las interacciones entre el trabajo, su medio, la satisfacción en el trabajo, la organización las capacidades del trabajador, sus necesidades, la cultura y la situación personal fuera del trabajo, en el caso de los trabajadores operativos participantes estos factores están presentes y a través del discurso que brindaron se lograron ejemplificar (Juárez. 2007, p. 58).

Además, Juárez (2007) en su estudio explica que en una organización no basta con quedarse solamente en los términos laborales sino que además se debe incluir los factores psicosociales por que estos permiten a la organización un análisis integral de su constitución y funcionamiento y que términos considerar para alcanzar un desarrollo organizacional óptimo.

Asimismo, Juárez (2007, p. 58) en su estudio determinó dos dimensiones que se presentan en el contexto laboral y que se relacionan con los factores psicosociales

a) Estresores para el trabajador que surgen de factores del ambiente físico y social, estos pueden ser: carga del trabajo, falta de autonomía y apoyo del jefe.

En el caso de los trabajadores municipales -como se explicará más adelante- no se presentan estresores como los menciona el autor, no obstante, la falta de autonomía es unos de las temáticas que mencionaron en su discurso, el cual se considera como un factor que interviene en la satisfacción laboral.

b) Afrontamiento: como los individuos perciben su entorno y cómo reaccionan ante el mismo.

En el caso de los trabajadores municipales la forma en la que afrontan su ambiente laboral se refleja en las siguientes frases extraídas de sus discursos:

A: "la cara de la municipalidad somos nosotros, por nosotros están limpias las calles"

$\mathrm{C}$ : "les he dicho a mis hijos ¿qué sería del mundo sin enterradores? Me he puesto a pensar que soy importante porque estoy ayudando a la gente en un momento difícil" 
D: "gracias a Dios la gente es cochina y tira basura, por medio de esas personas yo tengo mi trabajito, gracias a nosotros las calles están limpias"

E: “(...) el trabajito que hago es importante para las personas”

De acuerdo con las frases, el afrontamiento de estos trabajadores remite a que perciben su trabajo positivamente y lo consideran importante, lo cual les permite seguir realizando sus labores diarias por que creen en lo que hacen tiene un valor importante para los demás y para ellos mismos.

Por su parte, Gil-Monte (2012) explica que los factores psicosociales contribuyen positiva o negativamente en la actividad laboral y en la calidad de vida del trabajador. Respectivamente, el primero fomenta el desarrollo personal de las personas y el segundo cuando el desarrollo es desfavorable se promociona la salud y el bienestar para que este no sea vea perjudicado.

Por lo tanto, el considerar que el individuo no se puede estudiar aisladamente de su contexto social, familiar, laboral, educativo, entre otros; en la presente investigación involucrar los factores psicosociales con la satisfacción laboral ha permitido comprender y romper con algunas etiquetas impuestas socialmente y además acercarse a la realidad laboral que vivencia estos trabajadores, tal y como lo expresa " $\mathrm{A}$ " en su discurso "la gente ve los estudios que uno tiene y dice ¡esa! (...) la gente puede decir que uno es barredor pero con estudios" sumando la etiqueta construida socialmente que menciona "A" la gente cree que uno es un analfabeto" ante estos ejemplos, haber considerado la historia laboral antes de ingresar a la Municipalidad permitió romper esquemas de pensamiento estereotipados y mostrar que hay una historia, una razón que los ha colocado en ese puesto.

Por su parte, la organización como un sistema social conformada por un grupo de personas facilita un espacio donde se dan procesos sociales que influyen en el individuo y por ende en los ambientes de trabajo, por lo que el aspecto psicosocial articula lo estructuralorganizacional con las individualidades de las personas y de la organización misma por lo que no se pueden aislar una de otra para ser estudiadas (Palací, 2008).

En base a lo anterior, la organización ha implantado una cultura y comportamiento organizacional que ha nutrido una serie de aspectos psicosociales que ha afectado a los trabajadores (Chiavenato2004; Furham, 2009), en palabras de ellos se mencionan algunas frases:

A: “(...) a nivel jerárquico sabemos que somos la parte baja... desvalorizan el trabajo” 
B: "Aquí nadie valora el trabajo"

C: "Uno se siente como en una burbuja encerrado, no nos toman en cuenta, yo me siento aislado..."

D: “(...) si lo haces bien no dicen nada, si lo haces mal te dicen que porque tan cochino, que aquí y allá"

Esta cultura ha ocasionado que los trabajadores perciban que la Municipalidad los ve como "lo menos" de la institución y ha generado en los empleados frustración y falta de motivación por parte de la institución:

A: "la municipalidad no da motivación..."

B: "aquí nunca nadie valora el trabajo, aquí nunca nadie le llega a decir a uno que bien hizo el trabajo o la felicito o gracias... espero un gracias pero aquí no"

C: "gané mucha experiencia en Pipasa y aquí no la estoy aprovechando, aquí nada más es vaya haga un hueco y cállese... el carisma que tenía me lo apagaron"

D: “aquí no hay motivación por nada

De acuerdo a lo mencionado, los trabajadores esperan de la organización un excelente lugar para trabajar, reconocimiento, oportunidades de crecimiento, autonomía, compañerismo y calidad de vida laboral (Chiavenato, 2004, p. 30). En el caso de los trabajadores algunos demandan poder recibir parte de estos elementos por que en los años de trabajo no los han recibido o los tuvieron y se los quitaron, lo cual ha afectado la motivación extrínseca (Peiró y Prieto, 2007) que pueden recibir por parte de la organización y han continuado en la institución por su propia motivación, sus ideales y metas proyectadas en un futuro.

En cuanto, al ambiente de trabajo fuera de la organización, es decir, cómo los ven los usuarios y familiares, algunos indicaron haber enfrentado comentarios de mal gusto al mismo tiempo recibido motivación por parte de las personas externas de la institución. A través del discurso de los participantes se corroboró las etiquetas sociales que se han construido, tales como:

A: "nos ven como analfabetos... nos tratan como si no valiéramos nada"

\section{pesco


B: "para ellos soy el patito feo, me han menospreciado, humillado... para eso le pagan para que barra...la gente es tremenda"

C: "una persona me dijo enterrador, burlándose de mi...la gente es muy cruel... siento que mi hija se avergüenza de mi..."

D: "la gente cree que un barredor de calles es la persona más despreciable, nos dicen vagos, nos han tratado como una caca..."

E: "me han dicho le pagan para estar de vago y he tenido que explicarle la razón por la que me siento, por mi condición de salud"

Con base a lo anterior, estas etiquetas sociales responden a esos prejuicios, discriminaciones y estereotipos que se les ha puesto por su condición de trabajo y no por su condición de persona, es decir, que socialmente se considera que una persona es por el tipo de trabajo que realiza dejando de lado que sé es persona ante todo y el trabajo es un complemento del desarrollo de vida y no define como tal a la persona (Vidal, 2002).

Aunado a lo anterior, la misma organización ha nutrido estos estigmas sociales a través de la cultura, el comportamiento organizacional, la motivación al trabajador, ubicándolos como trabajadores desvalorizados. Los mismos empleados son conscientes de la imagen que los demás -incluyendo la organización- tienen de ellos, han sido capaces de reconocer las emociones positivas y negativas que producen en los demás, calificando su desempeño según como los ven los otros, por lo que influye en como ellos perciben su propio trabajo (Alvarado y Garrido, 2004). Todos los trabajadores participantes consideran su trabajo importante y valioso, algunos ejemplos son:

A: "la cara de la municipalidad somos nosotros, por nosotros están limpias las calles"

C: "les he dicho a mis hijos ¿qué sería de este mundo si no hubiera enterradores?, me he puesto a pensar que soy importante porque estoy ayudando a la gente en un momento difícil”

D: "gracias a Dios que la gente es cochina y tira basura, por medio de esas personas yo tengo mi trabajito, gracias a nosotros las calles se mantienen limpias"

E: "mi esposa se dio cuenta al visitarme que el trabajito que hago que es importante para las personas"

Con respecto a las implicaciones emocionales que se presentan en los trabajadores por el tipo de trabajo que realizan son: frustración, desvalorización, desmotivación por parte 
de la institución, humillaciones y satisfacción laboral. Todos estos elementos provienen tanto del ambiente de trabajo, como de la familia y de los usuarios. Para enfrentar todas estas situaciones algunos se quedan callados, otros dan explicaciones, las ignoran, o bien no hacen nada y siguen cumpliendo con su labor.

A: "todo lo bueno que hagamos algún día será recompensado, el esfuerzo me lo recompensa Dios y no el hombre, buscaré una oportunidad en otro lugar"

B: "el que persevera alcanza, hago mi trabajo lo mejor que puedo"

C: "aunque no se esté feliz y pasen cosas feas hay que echar para adelante..."

D: "este trabajo me da beneficios que en otro lugar no encuentro hay que seguir por la familia de uno"

E: "hay que hacer el trabajito bien, hay de todo en la viña del señor, a mis 35 años no he tenido problemas con el jefe ni con mis compañeros, la gente me quiere mucho"

Por lo tanto, la satisfacción laboral vinculada con las implicaciones psicosociales determinadas en los trabajadores proviene de que al ser empleados de un gobierno local obtienen una serie de beneficios como lo son: anualidad, vacaciones, bono escolar, un horario laboral que permite pasar más tiempo con la familia, un trabajo que consideran no es duro, es decir, obtienen una serie de beneficios que consideran que en otro lugar no encontrarían.

Asimismo, la satisfacción laboral al referirse a la actitud que tienen los trabajadores hacia su trabajo (Peiró y Prieto, 2007), los participantes manifiestan que se sienten bien con su labor y agradecidos por el hecho de tener trabajo pues algunos por la historia laboral que han tenido han caído a un puesto que les ha brindando la estabilidad laboral que han buscado, algunos les ha permitido crecer profesional y personalmente y a otros no perder la esperanza de encontrar un ascenso o una mejor oportunidad de trabajo. Cabe destacar que algunos de ellos como es el caso de "B" y de "E" esperan salir pensionados de la institución lo cual les motiva y les satisface.

En correspondencia al completar la frase "Ser trabajador municipal significa..." los trabajadores crearon el siguiente significado:

"Ser trabajador municipal significa dinero, amor al trabajo, trabajar con excelencia, forma parte de vida y es lo mejor que puede haber"

\section{peso


Con base a lo anterior, para la presente investigación se toma el discurso del trabajador como la principal técnica de recolección basada en la hermenéutica-dialéctica y respetando el sentir y pensar del trabajador (Álvarez, 2007) ante esto se determina lo siguiente:

a) Intención: la filosofía del trabajador municipal radica en que trabajar en una institución del gobierno le brinda estabilidad laboral y una serie de incentivos que en otro lugar no encontraría. Asimismo la actitud hacia su trabajo es de su agrado pues obtiene beneficios y a partir de la historia laboral han llegado a este puesto por el requerimiento y se han mantenido por lo antes mencionado.

b) Significado: frente a todos los factores psicosociales que se les presente en su ambiente laboral, los trabajadores lo han tomado como situaciones que forman parte del trabajo que se les presenta en su ambiente laboral, los trabajadores lo han tomado como situaciones que forman parte del trabajo que hay personas que son crueles pero a su vez ha habido personas muy buenas con ellos.

c) Función: hay una necesidad grande por hablar y ser escuchado en relación a las situaciones que han vivido durante sus historia de trabajo laboral, algunos con lagrimas otros con uno tono de voz cortado, implícitamente se identifica la importancia de trabajar a nivel psicológico con estas personas.

d) Ambiente y cultura: la cultura y comportamiento organizacional ha nutrido la brecha social que se ha creado hacia estos trabajadores a partir de la desvalorización del trabajo. Por su parte, las personas externas a la institución ha atribuido prejuicios y discriminación hacia estos trabajadores construyendo mitos y estereotipos que no corresponden a la realidad de lo que son y viven estos trabajadores. a pesar de estas circunstancias los trabajadores visualizan su trabajo como importante y sienten agrado por él.

A modo de reflexión, partiendo de la percepción de los trabajadores los factores psicosociales implicados en el trabajo se vinculan con la satisfacción laboral en tanto ellos mantengan una actitud positiva y un agrado por las labores que realizan para la Municipalidad.

Se consideran que son personas resilientes que a pesar de las dificultades que han pasado a través de su historia laboral han salido adelante y han demostrado que la estigmatización social construyen mitos pero no realidades. 
Aunado a lo anterior Furham (2009) describe que el trabajo significa para las personas un lugar de permanencia al que le dedican tiempo y esfuerzo, por lo que crear un equilibrio entre lo que desea el trabajador y la Municipalidad podría permitir relaciones humanas, oportunidades de crecimiento, compañerismo, comunicación asertiva y un clima organizacional agradable, eficiente y productivo con capacidad de brindar a los usuarios calidad de servicio, pues si la Municipalidad a lo interno tiene a sus empleados en optimas condiciones se proyectará a los usuarios con un desempeño de calidad.

En base a todo lo mencionado claro está que el uso del relato resulto ser un medio de aproximación a la realidad laboral, de un rompimiento de etiquetas sociales, de conocer la percepción de la organización desde la voz y experiencia de los mismos trabajadores y así vincular los factores psicosociales con la satisfacción laboral. Según Bruner (1998) citado por Mantilla, Ramírez y Arcila (2007) contar historias -en este caso historias laboralespermite hacer una descripción de la realidad, el acto de narrar permite construir según el entorno que rodea al individuo y precisamente eso fue lo que se logró con la participación de los cinco trabajadores.

Finalmente, la organización es un sistema social que reúne una serie de personas para cumplir objetivos y metas laborales, pero al facilitar un espacio para procesos e interacciones sociales lo convierte en una institución con valores, creencias, con una cultura propia, por lo que debe brindar a sus trabajadores una calidad de vida laboral integra, impulsarlos a lograr los objetivos y metas institucionales con un trato humano y adecuado, sensibilizar a la Municipalidad (todos sus miembros) para romper con las brechas sociales y hacer del ambiente laboral un lugar agradable, motivador, de pertenencia (Furham, 2009; Chiavenato, 2004), ya que al final no solo se benefician los trabajadores sino la organización misma, pues está generando estrategias que le brindaran eficiencia, calidad y productividad.

\section{Conclusión}

Ante el planteamiento del problema ¿Qué implicaciones psicosociales se presentan en el quehacer diario de estos cinco trabajadores? se encontraron: la frustración, la desvalorización del trabajo, predominancia de prejuicios y discriminaciones por el puesto que ocupan, falta de motivación por parte de la organización, oportunidades de ascenso nulas y participación en las decisiones organizacionales limitadas.

\section{esco


En lo que corresponde a si ¿existe satisfacción laboral en estos trabajadores que realizan labores que han sido desvalorizados socialmente? se podría afirmar que si hay satisfacción laboral basándose en la aproximación teórica que se le ha otorgado a este concepto, pues los trabajadores indicaron estar agradecidos y satisfechos de tener el trabajo que tienen porque este les ha permitido suplir algunas necesidades personales y de estudio para salir adelante vinculando este resultado con la historia laboral que han experimentado pues el trabajo actual les brinda estabilidad y beneficios por ser funcionarios públicos.

De acuerdo con el proceso realizado se logró identificar que los trabajadores demandan ser escuchados y de ser tratados como personas, por lo que esperan que la organización les brinde un trato más humanitario y menos despectivo ya que desde su visión de mundo no lo han percibido.

Asimismo, se logró identificar los factores psicosociales presentes en estos cinco trabajadores a partir del trabajo que realizan, los factores de mayor relevancia son: la desvalorización, falta de motivación por parte de la Municipalidad, los prejuicios y denigración por el tipo de trabajo que realizan, dificultad para ascender de puesto, oportunidad de crecimiento personal y profesional.

Por su parte, los trabajadores perciben que su trabajo es importante lo cual implica que tengan una actitud positiva respecto al quehacer diario, contribuyendo así a la existencia de una satisfacción laboral que promueve razones para permanecer en el puesto durante años o bien replantearse la idea de empezar a buscar oportunidades de crecimiento laboral.

Otro aspecto relevante durante la investigación, fue que los participantes aprovecharon el espacio para contar su historia laboral de una forma veraz, es decir, cada trabajador estuvo anuente a contar su historia. Algunos mencionaron situaciones muy personales, otros se dedicaron únicamente hablar del trabajo que realizan, pero se reflejó en el discurso la necesidad de hablar de la situación dentro de la Municipalidad así como la necesidad de escucha. Es importante destacar que la institución no cuenta con un profesional en psicología que cubra estas necesidades que presentan los trabajadores.

Se considera que esta investigación permitió una aproximación a la realidad en la cual se desenvuelven estos trabajadores, por lo que la articulación de la psicología social aplicada a las organizaciones permitió que esto fuera posible para concebir el sentir y pensar de los empleados dentro del gobierno local así como sus expectativas y realidades como funcionarios y como personas. 
Por último, se logró a través de esta investigación romper algunos estigmas sociales que se han construido hacia estas personas como lo es la etiqueta de "analfabetos" pero al indagar en su historia antes de ingresar Aseo de Vías y los primeros años de trabajo se encontró que la Municipalidad cuenta con una criminóloga, una auxiliar de enfermería y un administrador de agencia, lo cual implica que la estigmatización social construye mitos pero no realidades.

Finalmente, estos cinco trabajadores permitieron conocer a profundidad sus historia de vida laboral con la mayor veracidad posible y relatando situaciones que los ha marcado emocionalmente por lo que la información brindada tienen una nivel de confiabilidad alta.

\section{Referencias}

Alvarado, J y Garrido, A. (2004). Psicología Social: Perspectivas Psicológicas y Sociológicas. España: McGraw-Hill.

Alvarado, J; Zúñiga, M. (2012). Diagnóstico de Clima Organizacional (Informe diagnóstico). Heredia, Costa Rica.

Álvarez, J. (2007). ¿Cómo hacer investigación cualitativa? Fundamentos y metodología. $2^{\circ}$ edición. España: Paidós

Barrantes, R. (2007). Investigación: un camino al conocimiento. Un enfoque cuantitativo y cualitativo. San José, Costa Rica. EUNED.

Chiavenato, I. (2004). Comportamiento Organizacional. México. Thomson.

Furham, A. (2009). Psicología Organizacional. El comportamiento del individuo en las organizaciones. México: ED Alfaomega.

Gil-Monte, P. (2012). Riesgos psicosociales en el trabajo y salud ocupacional. Revista Peruana de Medicina Experimental y Salud Pública. 29 (2), pp. 237-241. Recuperado de: http://eds.a.ebscohost.com.una.idm.oclc.org/ehost/detail?sid=b659b51f-2b094095-bf04-

f07c99883cef\%40sessionmgr4004\&vid=1\&hid=4202\&bdata=Jmxhbmc9ZXMmc21 0ZT1laG9zdC1saXZl\#db=a9h\&AN=92944595

González, M y Olivares, S. (2009). Psicología del Trabajo. México: Grupo Patria.

\section{psico


Guillén, C; Guil, R. (2003). Psicología del Trabajo para las relaciones laborales. Universidad de Cáliz, España. De McGraw Hill.

Hernández, R; Fernández, C. y Baptista, P. (2003). Metodología de la Investigación. México: Mc Graw Hill.

Hogg, V. (2010). Psicología Social. Madrid, España: ED Panamericana.

Juárez, A. (2007). la 9 (1), pp- 57-64. Recuperado de: http://eds.a.ebscohost.com.una.idm.oclc.org/ehost/pdfviewer/pdfviewer?sid=4284ff 1d-216e-486d-af54-54a36f60fce0\%40sessionmgr4005\&vid=2\&hid=4202

Mantilla, F; Ramírez, A y Arcila, G. (2007). Instituciones del Estado y Vida Cotidiana: significados construidos por los funcionarios. Universitas Psychologica. 6 (1), pp. 173-183. Bogotá, Colombia. Recuperado de: http://eds.b.ebscohost.com.una.idm.oclc.org/ehost/detail?vid=3\&sid=ab0b5e0b2b05-495a-bbf0061e28a9140d\%40sessionmgr113\&hid=102\&bdata=Jmxhbmc9ZXMmc210ZT1laG $9 \mathrm{zdC} 1 \mathrm{saXZl} \# \mathrm{db}=\mathrm{a} 9 \mathrm{~h} \& A \mathrm{~N}=31169962$

Muchinsky, P. (2007). Psicología Aplicada al Trabajo. España: ED. Thomson.

Palací, F. (2008). Psicología de la Organización. España: Pearson Prentice Hall

Peiró, J; Prieto, F. (2007). Tratado de Psicología de Trabajo. Barcelona, España.

Vega, H. (2013). Investigación Cualitativa para el abordaje de la Gestión Pública Local. 6 (11). Revista Venezolana Observación Laboral. Universidad de Carabobo Venezuela. Extraído de: http://servicio.bc.uc.edu.ve/faces/revista/lainetv6n11/art04.pdf

Vidal, P. (2002). La identidad estigmatizada. Revista Universidad Bolivariana. 1 (3). Chile. Recuperado de: http://redalyc.uaemex.mx/pdf/305/30510309.pdf. 